\title{
Small for Gestational Age Births, Gestational Age, and Labour Outcomes: A Population-Based Study in the US
}

\author{
Justin Brandt ${ }^{1}$ and Cande Ananth (STATS CONSULTS ONLY) ${ }^{1}$ \\ ${ }^{1}$ Rutgers Robert Wood Johnson Medical School
}

March 14, 2021

\begin{abstract}
Objective: To estimate the causal impact of small for gestational age (SGA) births on caesarean delivery, with and without trial of labour (TOL); and to quantify how much of the association is mediated through gestational age at delivery. Design: Cross-sectional analysis. Setting: Para 2 women who delivered non-anomalous, singleton live births from 22-44 weeks' gestation in the US (2015-2018). Main outcomes and measures: Caesarean delivery with and without TOL. The exposure was SGA births (sex-specific birthweight $<5$ th and $<3$ rd percentiles for gestational age), and AGA births (10-89th percentile). We performed causal mediation analysis to determine the impact of gestational age at delivery (22-33, 34-36, 37-38, 39-40 and [?]41 weeks) as intermediate. Results: Of the 3,755,798 subjects, compared to AGA (29.6\%), caesarean risks were higher for SGA <5th $(34.3 \%)$ and SGA $<3$ rd $(36.4 \%)$ percentiles. For SGA $<5$ th percentile, the adjusted excess risk of caesarean delivery without TOL had a "U" shaped association, with increased risk at preterm gestations, nadir at 39-40 weeks, and increased thereafter. The decomposition analysis revealed the driver of this excess risk was SGA births. The risk of caesarean delivery with TOL was highest $<34$ weeks' gestation and was primarily an interaction effect. As gestation advanced, SGA births contributed proportionately greater to the risk. Associations were stronger for SGA <3rd percentile. Conclusions: Exposure to SGA drives high rates of prelabour caesareans and contributes to high risks of caesarean deliveries after TOL at $>41$ weeks gestation; a different mechanism drives high rates of caesareans after TOL at preterm gestations.
\end{abstract}

\section{Introduction}

Caesarean deliveries are associated with more maternal morbidity than vaginal deliveries, yet more than one-third of pregnancies in the United States (US) are delivered by caesarean (1). Various factors contributed to the caesarean epidemic, including maternal and fetal indications, labour management, and nonmedical factors (1). Small for gestational age (SGA), a proxy for fetal growth restriction that includes both constitutionally small and pathologically growth restricted fetuses, is one such fetal indication $(2,3)$. For pathologically growth restricted fetuses, transient contraction of the placental intervillous space during labour induces hypoxic-ischemic stress that may be poorly tolerated, resulting in category 2 fetal heart rate tracings, reflecting "fetal distress" in labour $(4,5)$. While some fetuses with severe growth restriction may not tolerate labour, an indication for caesarean delivery (6), others will have normal fetal heart rate patterns. In order to avoid emergent delivery for potential labour complications, obstetricians may encourage some patients to undergo prelabour caesarean deliveries. This counseling, which may be impacted by gestational age, often leads to management decisions that remain unsupported by empirical evidence and evidence-based recommendations (1).

SGA is associated with labour outcomes, including caesarean delivery, regardless of trial of labour (TOL) (7). Gestational age influences this relationship, but the extent to which gestational age impacts labour outcomes remains unknown. Therefore, we undertook this population-based study to determine if gestational age mediates or interacts with exposure to SGA to influence the risk of caesarean delivery. We performed a causal mediation analysis, which is an analytical method that provides a causal framework to disentangle 
the impact of gestational age as mediator, interaction, or both on exposure to SGA and labour outcomes (8-12). We hypothesized that gestational age would have the largest impact on labour outcomes $<28$ weeks' gestation and, particularly, for severely growth restricted fetuses.

\section{Methods}

Study design and data sources

We performed a cross-sectional analysis of non-anomalous, singleton live births who were delivered in the US from 2015-2018 using natality data assembled by the National Center for Health Statistics (NCHS). These data are collected by delivery attendants and transferred to the NCHS where they are cleaned, compiled, and de-identified. Demographic characteristics, medical and health information, and newborn outcomes were derived from the 2003 revision of the US standard certificate of live birth. All data used in this study are publicly available in de-identified form, and Institutional Review Board approval was therefore not sought. The study followed the STROBE reporting guidelines for cross-sectional studies.

The study population included women who delivered non-anomalous, singleton live births between 22-44 weeks' gestation. We restricted the analysis to para 2 women to avoid confounding by number of prior caesarean deliveries.

Exposure

The exposure was SGA, defined as sex-specific birthweight $<5^{\text {th }}$ and $<3^{\text {rd }}$ percentiles for gestational age based on a previously established US birthweight reference for singleton live births (13). We excluded large for gestational age births (i.e., $90^{\text {th }}$ percentile or higher) since these fetuses may have different thresholds for labour tolerance and caesarean delivery risks compared to SGA births. Infants with birthweight-for-gestational age between the $10-89^{\text {th }}$ percentiles constituted the appropriate for gestational age (AGA) reference group.

\section{Primary and secondary outcomes}

We had two primary outcomes. The first primary outcome was caesarean delivery after TOL. This outcome includes caesarean deliveries performed for fetal indications, such as category 2 fetal heart rate tracings (5) during labour. The second was caesarean delivery without TOL. Given that the study was limited to women with live born parity 2 , this outcome includes women with one prior vaginal delivery who may have undergone a prelabour caesarean to minimize the risk of labour complications.

\section{Gestational age as mediator}

Gestational age impacts labour outcomes for SGA pregnancies (14-17) and, thus, was evaluated to determine its impact as an intermediate in this analysis. Gestational age (in completed weeks) was derived from the best obstetrical estimate of the infant's gestation (18). We analyzed the distribution of gestational age as extremely preterm (21-27 weeks), very preterm (28-31 weeks), moderately preterm (32-33 weeks), late preterm (34-36 weeks), early term (37-38 weeks), term (38-40 weeks), late-term (41 weeks), and post-term (42-44 weeks) (19).

\section{Cohort composition}

Of the 15,550,961 total live births in the US between 2015-2018, we sequentially excluded pregnancies to primiparous women and women of parity [?]3, multiple gestations, newborns with major malformations, missing gestational age, 886 with gestational age $<22$ weeks, and 120 with gestational age [?]45 weeks, as well as large for gestational age births. We additionally excluded pregnancies with missing data for TOL and previous caesarean delivery. After all exclusions, 3,755,798 non-anomalous, singleton live births delivered at 22-44 weeks remained in the cohort (eFigure 1 ).

\section{Statistical analyses}

We undertook causal mediation analyses to examine the effects of SGA on caesarean delivery with and without TOL. The analyses were performed for all subjects in toto, but we also performed separate analyses 
for women with and without one prior caesarean delivery. The results of these stratified analyses (eTables 3-8 in the Supplement) were similar to the overall analysis, so we have presented the overall analyses here.

The effects of exposure and mediator on caesarean delivery were estimated from a multiplicative scale (loglinear Poisson regression models with a log-link function and robust variance, with risk ratio $[\mathrm{RR}]$ as the effect measure) and an additive scale (log-linear Poisson regression models with an identity-link function and robust variance, with the risk difference $[\mathrm{RD}]$ as the effect measure). We undertook a causal mediation analysis based on a counterfactual framework $(11,20)$ to disentangle the total effect of the SGA-caesarean association that could be attributable to a pure mediation effect by gestational age, to an interaction effect (by gestational age), and no interaction or mediation effects $(21,22)$. This method decomposes the total effect of SGA on caesarean delivery (exposure-outcome association) in the presence of gestational age (mediator) with which the SGA (exposure) may interact. The resulting 4-way decomposition includes: (i) effect of SGA on mode of delivery in the absence of the mediator (i.e., at 39-40 weeks [reference]); (ii) interaction effect when the mediator is left to what it would be in the absence of the SGA exposure (i.e., among AGA births); (iii) mediated interaction; and (iv) pure effect mediated through gestational age. Stated differently, these 4 effects correspond to the portion of the SGA-caesarean delivery association that is neither due to mediation or interaction with gestational age (also referred to as the controlled direct effect [CDE]), just to interaction but not mediation (referred to as the reference-interaction $\left[\mathrm{INT}_{\mathrm{REF}}\right]$ ), to both mediation and interaction (referred to as the mediation-interaction [ $\left.\mathrm{INT}_{\mathrm{MED}}\right]$ ), and to just mediation but not interaction (referred to as the pure indirect effect [PIE]). The sum of these 4 decomposed effects amount to $100 \%$ on the RD scale.

To facilitate easier interpretation of causal effects, we focus on the proportion of the SGA-caesarean delivery association that is directly attributed to exposure to SGA (i.e., the CDE) and two other measures in the primary analysis: proportion of the SGA-caesarean delivery association that is mediated through gestational age and the proportion attributable to an interaction between SGA and gestational age (22). The full analysis of the 4-way decomposition is shown in eTables 3-8 in the supplement. 95\% confidence intervals (CI) for all of causal estimates were derived from the bootstrap resampling method with 2000 replications.

\section{Confounders}

The choice of confounders were guided by directed acyclic graph (DAG) (Figure 1 ). These included maternal age $(<15,15-19,20-24,25-29$ [reference], 30-34, 35-39, and [?]40 years), education (below high school, up to high school, college, and beyond college educated), maternal race/ethnicity (non-Hispanic White, non-Hispanic Black, Hispanic, or other race), maternal smoking (non-smoker [reference], smoked before pregnancy only, initiated smoking during pregnancy, or smoked before and during pregnancy), single marital status, and pre-pregnancy body-mass index (BMI). BMI $\left(\mathrm{kg} / \mathrm{m}^{2}\right)$ was categorized as underweight $(<18.5)$, normal (18.5-24.9), overweight (25.0-29.9), obese (30.0-34.9), and morbidly obese ([?]35.0). Other confounders included chronic hypertension, pregestational diabetes, and year of delivery.

\section{Sensitivity analysis for unmeasured confounding}

Mediation methods are developed under strict no-unmeasured confounding assumptions $(22,23)$. To examine the robustness of causal effects to unmeasured confounders, we estimated the E-value $(24,25)$. The E-value provides an estimate (on the excess risk scale) of the smallest excess risk of the unmeasured confounder(s), beyond that accounted for the adjusted confounders, to draw the observed excess toward the null. In addition, the E-value provides an indication of the smallest excess risk of the unmeasured confounder to move the lower $95 \%$ CI of the observed excess risk to cross the null.

All analyses were conducted using SAS, version 9.4 (SAS Institute, Cary, NC).

\section{Results}

During the four-year period (2015-2018), there were 3,755,798 para 2 women who delivered non-anomalous, singleton live-births at 22-44 weeks' gestation in the US (eTable 1 ). The overall caesarean rate was 29.9\%, and more than two-thirds of these caesareans were performed prelabour. Compared to AGA births (29.6\%), 
the caesarean delivery rates were higher for SGA $<5^{\text {th }}$ percentile $(34.3 \%)$ and SGA $<3^{\text {rd }}$ percentile $(36.4 \%)$. The cohort was predominantly college educated, normal weight Caucasian women.

Caesarean delivery without TOL

Caesarean delivery without TOL risks were higher for SGA $<5^{\text {th }}$ percentile $(25.2 \%$ versus $23.7 \%)$ and SGA $<3^{\text {rd }}$ percentile $(26.2 \%$ versus $23.7 \%$; Table 1$)$ compared to AGA. A more nuanced picture emerges when this outcome is stratified by gestational age. For example, $77.5 \%$ of women who delivered at $<34$ weeks with SGA $<5^{\text {th }}$ percentile were delivered by caesarean without TOL compared to $48.3 \%$ of AGA births whereas the risk was substantially lower at 41 weeks and beyond (10.9\% versus $8.0 \%)$. Although risks of caesarean delivery without TOL decreased across gestational age for SGA and AGA births, the caesarean risks without TOL were higher for SGA $<5^{\text {th }}$ percentile at each gestational age strata except for 39-40 weeks' gestation (when $22.9 \%$ of SGA $<5^{\text {th }}$ percentile births were Caesarean without TOL compared to $24.5 \%$ of AGA births). These same trends, though more pronounced, were observed for SGA $<3^{\text {rd }}$ percentile births.

The results of the mediation analysis for caesarean delivery without TOL and exposure to SGA $<5^{\text {th }}$ percentile are described in Table 2 . For SGA versus AGA births, the adjusted excess risk of caesarean delivery without trial of labour had a "U" shaped association, with increased risk at preterm gestations, lowest risk at 39-40 weeks, and increased risk at [?]41 weeks. The decomposition analysis revealed that the driver of this excess risk was the CDE with minimal mediation and interaction components. The mediation analysis for this outcome and exposure to SGA <3rd percentile revealed similar, but more pronounced, patterns (Table $3)$.

Caesarean delivery after TOL

Compared to AGA births, caesarean delivery after TOL risks were higher for SGA $<5^{\text {th }}$ percentile $(9.1 \%$ versus $5.9 \%)$ and SGA $<3^{\text {rd }}$ percentile $(10.2 \%$ versus $5.9 \%$; Table 1$)$. For SGA $<5^{\text {th }}$ percentile, further stratification by gestational age reveals that the risks of caesarean delivery after TOL were higher for SGA $<5^{\text {th }}$ percentile births across each gestational age strata, but the highest risks were [?]41 weeks $(15.2 \%)$. The lowest risk occurred from 39-40 weeks' gestation, although SGA $<5^{\text {th }}$ percentile births had higher risks of caesarean delivery after TOL compared to AGA births (8.0\% versus $5.6 \%)$. Again, these trends were mirrored, but more pronounced, for SGA $<3^{\text {rd }}$ percentile births.

The adjusted excess risk of caesarean delivery with TOL also had a "U" shaped association (Table 2 ). The magnitude of the risk was highest $<34$ weeks' gestation where the driver of risk was primarily due to interaction. As gestation advanced, the CDE had proportionately greater effect and the interaction component played a smaller role. These trends were similar, but more pronounced, for SGA $<3^{\text {rd }}$ percentile births (Table 3 ).

\section{Sensitivity analysis}

The results of the sensitivity analysis are described in eTable 2. The E-values, both for the excess risk and the lower $95 \%$ CI, are substantially higher than the corresponding observed estimates. These results suggest that the causal mediation parameters are robust to unmeasured confounding.

\section{Discussion}

In this large cross-sectional study based on US births, we evaluated the impact of gestational age on delivery mode for pregnancies complicated by SGA. Because the gestational age when delivery becomes indicated may influence labour outcomes, we performed a causal mediation analysis to quantify the mediating and interaction effects of gestational age on exposure to SGA and delivery mode. The principal finding of this study is that the risk for caesarean delivery with TOL at term gestations and caesarean delivery without TOL across all gestations for SGA births is primarily attributable to exposure to SGA rather than mediation or interaction effects of gestational age.

The caesarean delivery without TOL outcome includes women who had prelabour caesareans. The intent of performing a prelabour caesarean for some obstetricians is to avoid impending complications as a consequence 
of labour, including category 2 fetal heart rate tracings that may precipitate an emergent caesarean delivery. In this analysis, caesarean rates without TOL were higher for SGA births at each gestational age strata except for 39-40 weeks' gestation. The decomposition analysis revealed that exposure to SGA by itself (and independent of gestational age) was the driver of this risk.

The caesarean delivery after TOL outcome includes those who required caesarean deliveries for abnormal fetal heart rate patterns and labour complications. The rates of caesarean delivery after TOL were higher for SGA births across gestational age strata, but the highest rates were observed [?]41 weeks' gestation. When these rates were highest at term gestations, exposure to SGA was the dominant driver of caesarean delivery risk.

Prior studies have shown high rates of caesarean delivery for SGA pregnancies. For example, in a study of 2885 non-anomalous, singleton, cephalic presenting SGA fetuses at 25-34 weeks in New York (1995-2003), the overall caesarean delivery rate was $57.9 \%$ (26). That study was limited by small size, but also did not stratify the analyses by gestational age ranges, caesarean history, or caesarean indications. As a result, a picture emerges of caesarean risk for SGA pregnancies that may fuel concerns about high rates of emergent deliveries. While the results of this study also found higher overall caesarean delivery rates from 22-44 weeks' gestation for SGA versus AGA pregnancies $\left(34.3 \%\right.$ for SGA $<5^{\text {th }}$ percentile and $36.4 \%$ for SGA $<3^{\text {rd }}$ percentile versus $29.6 \%$ for AGA), a more nuanced picture emerges when caesarean rates are broken into gestational age ranges. For example, there were extremely high rates $<32$ weeks' gestation (over $80 \%$ ), but the rates improved as gestational age advanced. This breakdown by gestational age provides improved insight into the caesarean risk for SGA pregnancies.

The results of this analysis also demonstrated that labour outcomes for SGA pregnancies are impacted by indication for delivery. The overall high caesarean rates for SGA fetuses in the US were largely driven by prelabour procedures. Approximately two-thirds of all Caesareans were classified as prelabour procedures, but the risk was highest $<32$ weeks' gestation. Further, Caesarean delivery rates without TOL were considerably higher at each gestational age strata compared to rates with TOL, except at [?]41 weeks' gestation. These findings give credence to apprehensions raised by the NIH Consensus statement "Preventing the First Caesarean Delivery," that "concern[s] about vaginal delivery coupled with relative indifference regarding the risks of Caesarean may lead to a decision that is not based on clinical evidence" (1). The results of this analysis suggest that too many patients in the US are not given the opportunity to have TOL and spontaneous vaginal delivery.

In fact, while the rates of Caesarean delivery after TOL are higher for SGA versus AGA pregnancies, the magnitude of risk is less than some providers may recognize. Overall rates of caesarean with TOL were $9.1 \%$ for SGA $<5^{\text {th }}$ percentile and $10.2 \%$ for SGA $<3^{\text {rd }}$ percentile versus $5.9 \%$ for AGA births. The caesarean with TOL risks were notably highest for SGA and AGA births after 41 weeks gestation. Also, the risks of caesarean delivery with TOL was lowest for SGA versus AGA births at 39-40 weeks' gestation and with the smallest risk difference at this gestation age range. These observations corroborate the main findings of the ARRIVE trial (27) that induction of labour in the $39^{\text {th }}$ week reduces Caesarean delivery rates.

The results of this mediation analysis suggest that different mechanisms drive the risk of caesarean delivery after TOL at preterm versus term gestations. At preterm gestations, interaction of SGA with gestational age has a larger effect on the risk, whereas exposure to SGA itself has a large effect at term gestations. Unfortunately, this dataset cannot explain how the interaction affect contributes to caesarean delivery after TOL rates in preterm gestations. It is possible that the fetal heart rate monitoring profile of preterm SGA fetuses could provide insight, but this has not been elucidated. A 2010 Cochrane review that sought to assess the effects of a policy of elective caesarean delivery versus expectant management for growth restricted fetuses could only identify 6 trials with 122 patients (28). The included studies were marred by recruitment problems, which limited the conclusions of the systematic review. The manifestation of gestational age-SGA interaction as it relates to fetal tolerance of labour at preterm gestations requires further evaluation.

Exposure to SGA alone (i.e., the CDE) explains increased risks of caesarean delivery after TOL for term and 
late-term SGA births. In this case, it is likely that the impact of pathologic growth restriction drives the risk and likely reflects underlying ischemic placental disease (IPD) $(6,29)$. IPD, which also includes preeclampsia and placental abruption, has been implicated in over $50 \%$ of all clinician-initiated preterm deliveries and may play a more substantial role in term gestations $(6,29)$.

The main strength of the study is the application of a causal mediation approach, a cutting edge statistical methodology (30), to evaluate the individual contribution of SGA and the impact of gestational age on caesarean risk. Many studies in the obstetrical literature inappropriately attempt to adjust for gestational age as a confounder, leading to biased associations since gestational age may be on the causal pathway for many adverse perinatal outcomes $(31,32)$. Using causal mediation analysis, the study results clarify the impact of gestational age as an intermediate on the SGA-labour outcome paradigm to elucidate the causal relationship between exposure and outcome.

We explicitly outlined the assumptions that we used to make causal claims in this mediation analysis (12). Further, these assumptions were assessed by a sensitivity analysis that evaluated the extent unmeasured confounding could bias our results. The E-values were 2-3 fold larger than the observed effect estimates, suggesting that it would take very large effect sizes of unmeasured confounders to nullify the associations, a situation that is unlikely. This methodological approach strengthens the study, and suggests robustness of the findings.

The study has some limitations. The data could not provide insight into spontaneous versus clinicianinitiated preterm deliveries. We wanted to assess labour outcomes, including abnormal fetal heart rate patterns, but this information is not readily available from the data source. In order to capture women who had caesarean deliveries for fetal indications after unsuccessful TOL, we had to include all women who were coded as having caesarean delivery after TOL. Some of these patients required caesarean delivery for labour dystocia as well as maternal indications. Although we were able to avoid confounding due to prior caesarean delivery by limiting the analytic cohort to women of live birth parity 2 , we recognize that our primary outcome has some limitations.

\section{Conclusions}

We have clarified the mediating and interaction effects of gestational age on labour outcomes for pregnancies complicated by SGA in this causal mediation analysis. The impact of gestational age on caesarean delivery without TOL was profound across gestation. Approximately two-third of all caesareans were performed prelabour. With appropriate counseling, providers may feel comfortable offering TOL to these patients, optimally at 39-40 weeks' gestation.

Rates of caesarean delivery are increased among SGA after a TOL compared to AGA births at preterm and term gestations, but the effect of gestational age is different. An interaction between gestational age and SGA drives the risk at preterm gestations whereas exposure to SGA alone, likely a consequence of IPD, drives this risk at term. These findings suggest different strategies are needed to maximize vaginal delivery rates for SGA pregnancies who undergo TOL, but it is likely that timing the delivery at term and avoidance of late term gestation would have positive impact.

\section{Acknowledgments : none}

Disclosures of interest : Both authors declare no support from any organization for the submitted work; no financial relationships with any organizations that might have an interest in the submitted work in the previous three years; and no other relationships or activities that could appear to have influenced the submitted work.

\section{Contribution of authorship:}

JB: conceptualization, writing - original draft

CA: conceptualization, methodology, statistical analysis, writing - review and editing, supervision 
Details of ethics approval : None

Funding: None

\section{References}

1. Spong CY, Berghella V, Wenstrom KD, Mercer BM, Saade GR. Preventing the first cesarean delivery: summary of a joint Eunice Kennedy Shriver National Institute of Child Health and Human Development, Society for Maternal-Fetal Medicine, and American College of Obstetricians and Gynecologists Workshop. Obstet Gynecol. 2012;120(5):1181-93.

2. Ananth CV, Brandt JS, Vintzileos AM. Standard vs population reference curves in obstetrics: which one should we use? Am J Obstet Gynecol. 2019;220(4):293-6.

3. Ananth CV, Vintzileos AM. Distinguishing pathological from constitutional small for gestational age births in population-based studies. Early human development. 2009;85(10): 653-8.

4. Martin CB, Jr. Normal fetal physiology and behavior, and adaptive responses with hypoxemia. Seminars in perinatology. 2008;32(4):239-42.

5. Macones GA, Hankins GD, Spong CY, Hauth J, Moore T. The 2008 National Institute of Child Health and Human Development workshop report on electronic fetal monitoring: update on definitions, interpretation, and research guidelines. J Obstet Gynecol Neonatal Nurs. 2008;37(5):510-5.

6. Ananth CV, Vintzileos AM. Maternal-fetal conditions necessitating a medical intervention resulting in preterm birth. Am J Obstet Gynecol. 2006;195(6):1557-63.

7. McCowan LM, Figueras F, Anderson NH. Evidence-based national guidelines for the management of suspected fetal growth restriction: comparison, consensus, and controversy. Am J Obstet Gynecol. 2018;218(2s):S855-s68.

8. Ananth CV, VanderWeele TJ. Placental abruption and perinatal mortality with preterm delivery as a mediator: disentangling direct and indirect effects. Am J Epidemiol. 2011;174(1):99-108.

9. Vanderweele TJ, Vansteelandt S. Odds ratios for mediation analysis for a dichotomous outcome. Am J Epidemiol. 2010;172(12):1339-48.

10. VanderWeele TJ. Bias formulas for sensitivity analysis for direct and indirect effects. Epidemiology. 2010;21(4):540-51.

11. Robins JM, Greenland S. Identifiability and exchangeability for direct and indirect effects. Epidemiology. $1992 ; 3(2): 143-55$.

12. Lee H, Herbert RD, McAuley JH. Mediation Analysis. JAMA. 2019;321(7):697-8.

13. Talge NM, Mudd LM, Sikorskii A, Basso O. United States birth weight reference corrected for implausible gestational age estimates. Pediatrics. 2014;133(5):844-53.

14. Joseph KS, Demissie K, Kramer MS. Obstetric intervention, stillbirth, and preterm birth. Semin Perinatol. 2002;26(4):250-9.

15. Metcalfe A, Lisonkova S, Joseph KS. The association between temporal changes in the use of obstetrical intervention and small-for-gestational age live births. BMC Pregnancy Childbirth. 2015;15:233.

16. Ananth CV, Vintzileos AM. Medically indicated preterm birth: recognizing the importance of the problem. Clin Perinatol. 2008;35(1):53-67.

17. MacDorman MF, Declercq E, Zhang J. Obstetrical intervention and the singleton preterm birth rate in the United States from 1991-2006. Am J Public Health. 2010;100(11):2241-7. 
18. Martin JA, Osterman MJ, Kirmeyer SE, Gregory EC. Measuring Gestational Age in Vital Statistics Data: Transitioning to the Obstetric Estimate. National vital statistics reports : from the Centers for Disease Control and Prevention, National Center for Health Statistics, National Vital Statistics System. 2015;64(5):1-20.

19. Spong CY. Defining "term" pregnancy: recommendations from the Defining "Term" Pregnancy Workgroup. JAMA. 2013;309(23):2445-6.

20. Pearl J. Direct and indirect effects. In: Kaufmann M, editor. Proceedings of the Seventeenth Conference on Uncertainty and Artificial Intelligence. San Francisco, CA2001.

21. Erratum: A Unification of Mediation and Interaction: A 4-Way Decomposition. Epidemiology. 2016;27(5):e36.

22. VanderWeele TJ. A unification of mediation and interaction: a 4-way decomposition. Epidemiology. 2014;25(5):749-61.

23. Valeri L, Vanderweele TJ. Mediation analysis allowing for exposure-mediator interactions and causal interpretation: theoretical assumptions and implementation with SAS and SPSS macros. Psychol Methods. 2013;18(2):137-50.

24. VanderWeele TJ, Ding P. Sensitivity Analysis in Observational Research: Introducing the E-Value. Ann Intern Med. 2017;167(4):268-74.

25. Mathur MB, Ding P, Riddell CA, VanderWeele TJ. Web Site and R Package for Computing E-values. Epidemiology. 2018;29(5):e45-e7.

26. Werner EF, Savitz DA, Janevic TM, Ehsanipoor RM, Thung SF, Funai EF, et al. Mode of delivery and neonatal outcomes in preterm, small-for-gestational-age newborns. Obstetrics and gynecology. 2012;120(3):560-4.

27. Grobman WA, Rice MM, Reddy UM, Tita ATN, Silver RM, Mallett G, et al. Labor Induction versus Expectant Management in Low-Risk Nulliparous Women. N Engl J Med. 2018;379(6): 513-23.

28. Grant A, Glazener CM. Elective caesarean section versus expectant management for delivery of the small baby. The Cochrane database of systematic reviews. 2001(2): Cd000078.

29. Ananth CV, Smulian JC, Vintzileos AM. Ischemic placental disease: maternal versus fetal clinical presentations by gestational age. The journal of maternal-fetal \& neonatal medicine : the official journal of the European Association of Perinatal Medicine, the Federation of Asia and Oceania Perinatal Societies, the International Society of Perinatal Obstet. 2010;23(8):887-93.

30. Imai K, Keele L, T Y. Identification, inference, and sensitivity analyses for causal mediation effects. Stat Sci. 2010;25(1):51-71.

31. VanderWeele TJ, Mumford SL, Schisterman EF. Conditioning on intermediates in perinatal epidemiology. Epidemiology. 2012;23(1):1-9.

32. Ananth CV, Schisterman EF. Confounding, causality, and confusion: the role of intermediate variables in interpreting observational studies in obstetrics. Am J Obstet Gynecol. 2017;217(2):167-75.

\section{Figure legends}

Figure 1 Simplified directed acyclic graph showing the relationship between exposure to SGA and labour outcomes with gestational age as the mediator: United States non-anomalous singleton live births, 2015-2018

Legend : Simplified DAG depicting the relationship between the small for gestational age (SGA) exposure and labour outcomes, including Caesarean delivery with and without trial of labour, in the presence of gestational age (mediator) and measured and unmeasured confounders. 


\section{Supplemental figure}

eFigure 1 Study flow diagram: United States non-anomalous singleton live births, 2015-2018

Table 1 Labour outcomes in relation to gestational age at delivery and birthweight-forgestational age percentiles

\begin{tabular}{|c|c|c|}
\hline Gestational age (weeks) & Total births & $\mathrm{Ca}$ \\
\hline Birthweight-for-gestational age $<5^{\text {th }}$ percentile & Birthweight-for-gestational age $<5^{\text {th }}$ percentile & Bir \\
\hline $22-44$ & $161,934(100)$ & 40,8 \\
\hline $22-33$ & $2789(1.7)$ & 216 \\
\hline $34-36$ & $11,432(7.1)$ & 488 \\
\hline $37-38$ & $55,733(34.4)$ & 13,4 \\
\hline $39-40$ & $86,346(53.3)$ & 19,7 \\
\hline 41 & $5634(3.5)$ & 614 \\
\hline Birthweight-for-gestational age $<3^{\text {rd }}$ percentile & Birthweight-for-gestational age $<3^{\text {rd }}$ percentile & Bir \\
\hline $22-44$ & $89,010(100)$ & 23,3 \\
\hline $22-33$ & $1493(1.7)$ & $117^{\prime}$ \\
\hline $34-36$ & $6740(7.6)$ & 306 \\
\hline $37-38$ & $31,324(35.2)$ & 797 \\
\hline $39-40$ & $46,330(52.1)$ & 10,7 \\
\hline 41 & $3123(3.5)$ & 379 \\
\hline Birthweight-for-gestational age $10-89^{\text {th }}$ percentile & Birthweight-for-gestational age $10-89^{\text {th }}$ percentile & Bir \\
\hline $22-44$ & $3,407,108(100)$ & 807 \\
\hline $22-33$ & $50,811(1.5)$ & 24,5 \\
\hline $34-36$ & $174,418(5.1)$ & \\
\hline $37-38$ & $845,106(24.8)$ & \\
\hline $39-40$ & $2,138,193(62.8)$ & \\
\hline 41 & $198,580(5.8)$ & \\
\hline
\end{tabular}

Table 2

Decomposition of the total effect of small for gestational age $<5^{\text {th }}$ percentile birth on caesarean delivery due to mediation and interaction with gestational age at delivery

Gestational age at delivery (weeks)

Total excess risk ratio $(95 \% \mathrm{CI})$

Controlled direct effect Excess risk ratio (95\% CI)

Attributed to mediation Percent (95\% CI)

Due to interaction

Percent (95\% CI)

Caesarean delivery without trial of labour

Caesarean delivery without trial of labour

Caesarean delivery without trial of labour

Caesarean delivery without trial of labour

Caesarean delivery without trial of labour 
$43.5(39.6,47.4)$

$89.4(86.7,91.9)$

$-17.3(-21.5,-14.0)$

$11.5(9.1,14.3)$

34-36

$53.5(49.7,57.6)$

$73.1(69.7,76.1)$

$3.2(2.5,4.1)$

$26.7(23.7,30.1)$

37-38

$53.3(49.9,56.3)$

$78.9(74.3,83.2)$

$3.2(2.3,4.2)$

$23.2(18.7,27.9)$

$39-40$

0.0 (Reference)

0.0 (Reference)

0.0 (Reference)

0.0 (Reference)

[?]41

$35.0(31.5,38.5)$

$101.8(98.1,106.8)$

$-8.7(-10.6,-7.0)$

$5.0(0.6,8.6)$

Caesarean delivery with trial of labour

Caesarean delivery with trial of labour

Caesarean delivery with trial of labour

Caesarean delivery with trial of labour

Caesarean delivery with trial of labour

$22-33^{*}$

$78.6(67.7,90.9)$

$10.7(7.6,14.2)$

$1.3(-3.3,5.7)$ 
$89.1(85.7,92.2)$

34-36

37.8 (34.0, 41.4)

$24.1(17.8,29.3)$

$22.5(20.4,24.7)$

$70.4(65.3,76.3)$

$37-38$

$24.3(21.9,26.7)$

$41.1(32.0,50.1)$

$13.9(11.8,16.2)$

$59.5(50.5,68.6)$

$39-40$

0.0 (Reference)

0.0 (Reference)

0.0 (Reference)

0.0 (Reference)

[?] 41

$10.5(7.4,13.3)$

$79.9(69.0,86.2)$

$9.9(7.3,14.1)$

$7.4(2.9,14.3)$

CI, confidence interval

All associations were adjusted for the confounding effects of year of birth, maternal age, maternal education, race/ethnicity, smoking, single marital status, and pre-pregnancy body-mass index

All 95\% confidence interval estimates were based on 2000 bootstrap samples

* The 4-way decomposition analyses stratified by gestational age ranges were similar at 21-27, 28-31, and 32-33 weeks so we grouped these gestational age ranges together

Table 3

Decomposition of the total effect of small for gestational age $<3^{\text {rd }}$ percentile birth on caesarean delivery due to mediation and interaction with gestational age at delivery

\begin{tabular}{|c|c|c|c|c|}
\hline $\begin{array}{l}\text { Gestational age } \\
\text { at delivery } \\
\text { (weeks) }\end{array}$ & $\begin{array}{l}\text { Adjusted excess } \\
\text { risk ratio }(95 \% \\
\text { CI })\end{array}$ & $\begin{array}{l}\text { Controlled direct } \\
\text { effect }(95 \% \mathrm{CI})\end{array}$ & $\begin{array}{l}\text { Attributed to } \\
\text { mediation \% } \\
(95 \% \text { CI })\end{array}$ & $\begin{array}{l}\text { Due to } \\
\text { interaction \% } \\
(95 \% \mathrm{CI})\end{array}$ \\
\hline $\begin{array}{l}\text { Caesarean } \\
\text { delivery } \\
\text { without trial of } \\
\text { labour }\end{array}$ & $\begin{array}{l}\text { Caesarean } \\
\text { delivery } \\
\text { without trial of } \\
\text { labour }\end{array}$ & $\begin{array}{l}\text { Caesarean } \\
\text { delivery } \\
\text { without trial of } \\
\text { labour }\end{array}$ & $\begin{array}{l}\text { Caesarean } \\
\text { delivery } \\
\text { without trial of } \\
\text { labour }\end{array}$ & $\begin{array}{l}\text { Caesarean } \\
\text { delivery } \\
\text { without trial of } \\
\text { labour }\end{array}$ \\
\hline
\end{tabular}




\begin{tabular}{lllll}
\hline $\begin{array}{l}\text { Gestational age } \\
\text { at delivery } \\
\text { (weeks) }\end{array}$ & $\begin{array}{l}\text { Adjusted excess } \\
\text { risk ratio } \mathbf{9 5 \%}\end{array}$ & $\begin{array}{l}\text { Controlled direct } \\
\text { effect }(\mathbf{9 5 \%} \text { CI) }\end{array}$ & $\begin{array}{l}\text { Attributed to } \\
\text { mediation \% } \\
\text { (95\% CI) }\end{array}$ & $\begin{array}{l}\text { Due to } \\
\text { interaction \% } \\
\text { (95\% CI) }\end{array}$ \\
\hline $22-33^{*}$ & $61.0(55.0,67.2)$ & $93.6(90.8,95.6)$ & $-12.7(-17.2,-9.1)$ & $7.2(5.2,9.9)$ \\
$34-36$ & $79.8(73.9,86.0)$ & $72.8(69.1,76.5)$ & $4.0(3.0,5.1)$ & $27.0(23.4,30.7)$ \\
$37-38$ & $79.7(74.6,84.4)$ & $78.6(74.0,83.4)$ & $3.9(2.8,5.0)$ & $23.0(18.1,27.6)$ \\
$39-40$ & 0.0 (Reference) & 0.0 (Reference) & 0.0 (Reference) & 0.0 (Reference) \\
{$[?] 41$} & $50.9(46.1,55.6)$ & $101.1(96.4,105.2)$ & $-5.6(-7.6,-4.2)$ & $3.2(-0.7,7.9)$ \\
Caesarean & Caesarean & Caesarean & Caesarean & Caesarean \\
delivery with & delivery with & delivery with & delivery with & delivery with \\
trial of labour & trial of labour & trial of labour & trial of labour & trial of labour \\
$22-33^{*}$ & $93.3(77.0,112.4)$ & $17.9(13.6,22.6)$ & $-1.0(-6.8,4.3)$ & $82.3(77.5,86.3)$ \\
$34-36$ & $59.4(54.0,65.0)$ & $29.5(24.4,34.4)$ & $23.3(21.1,25.8)$ & $66.0(61.2,71.0)$ \\
$37-38$ & $38.4(34.9,42.1)$ & $49.4(41.3,56.8)$ & $12.9(10.9,15.1)$ & $51.0(43.6,59.0)$ \\
$39-40$ & $0.0($ Reference) & $0.0($ Reference) & $0.0($ Reference) & 0.0 (Reference) \\
{$[?] 41$} & $19.8(15.6,24.0)$ & $88.5(82.5,93.2)$ & $4.3(2.9,6.1)$ & $5.4(1.4,10.1)$ \\
\hline
\end{tabular}

CI, confidence interval; CDE

All associations were adjusted for the confounding effects of year of birth, maternal age, maternal education, race/ethnicity, smoking, single marital status, and pre-pregnancy body-mass index

All 95\% confidence interval estimates were based on 2000 bootstrap samples

* The 4-way decomposition analyses stratified by gestational age ranges were similar at 21-27, 28-31, and 32-33 weeks so we grouped these gestational age ranges together

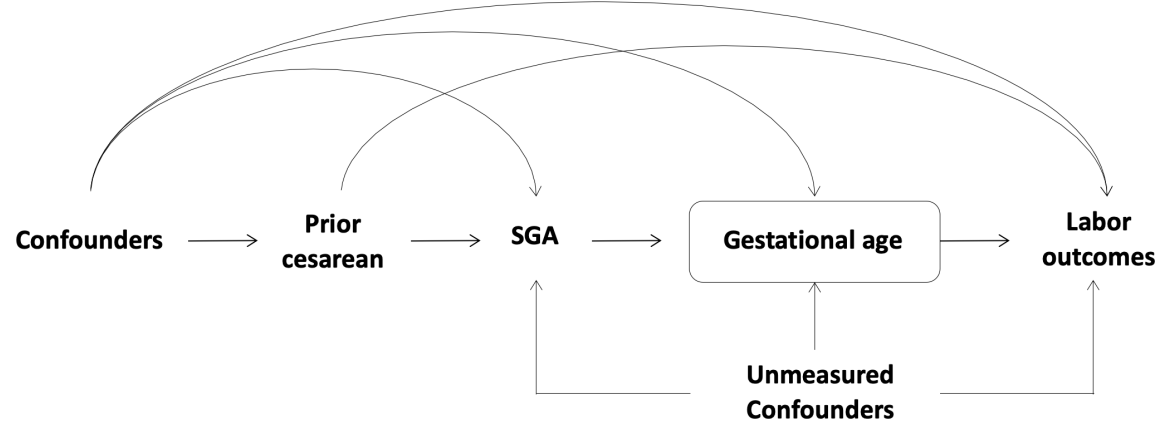


Total live births in the US, $2015-2018(n=15,550,961)$

$\begin{array}{ll}\rightarrow & \text { Parity } 1 \text { or parity } \geq 3 \text { ( } n=11,176,233) \\ & \rightarrow \text { Twins and higher-order births }(\mathrm{n}=148,285) \\ & \rightarrow \text { Major malformations }(\mathrm{n}=8138) \\ & \rightarrow \text { Gestational age missing }(\mathrm{n}=1614),<22(\mathrm{n}=886) \text { or } \geq 45(\mathrm{n}=120) \text { weeks } \\ & \text { Large for gestational age births }(\mathrm{n}=423,979) ; \text { missing SGA }(\mathrm{n}=6024) \\ & \text { Missing trial of labor }(\mathrm{n}=16,216) \text {, and previous cesarean }(\mathrm{n}=13,668) \\ & \text { Total exclusions ( } \mathrm{n}=11,795,163) \\ \text { Total non-anomalous, singleton, live births } \\ \text { delivered at 22-44 weeks }(\mathrm{n}=3,755,798)\end{array}$

